\title{
Effect of the Bone Morphogenetic Protein-2 Doses on the Osteogenic Potential of Human Multipotent Stromal Cells- Containing Tissue Engineered Constructs
} Authors: Adeline Decambron ${ }^{1,2,{ }^{*}}$, Nausikaa Devriendt ${ }^{3,{ }^{*}}$, Nathanael Larochette ${ }^{1,2}$, Mathieu Manassero ${ }^{1,2}$, Marianne Bourguignon ${ }^{1,2}$,
Hanane El-Hafci ${ }^{1,2}$, Hervé Petite ${ }^{1,2}$, Véronique Viateau ${ }^{1,2}$, and Delphine Logeart-Avramoglou

\author{
Affiliations: \\ 1 B2OA UMR 7052, Univ Paris Diderot, Sorbonne Paris Cité, CNRS, F-75010 Paris, France \\ 2 B2OA UMR 7052, Ecole Nationale Vétérinaire d'Alfort, Université Paris-Est, F- 94700 Maisons-Alfort, France \\ 3 Faculty of Veterinary Medicine of Ghent University, Salisburylaan 133. B-9820 Merelbeke, Belgium. \\ * Equal contribution (co-first author).
}

Key words: bone; tissue engineering; in vivo; BMP-2; MSC; dual delivery

ABSTRACT: The addition of bone morphogenetic protein-2 (BMP-2) with multipotent stromal cells (MSC) is an attractive strategy to enhance the bone-forming potential of MSC-based tissue engineering (TE) constructs. However, the effective dosage of BMP-2 remains to be determined. In this study, we evaluated the effects of human MSCs codelivered with BMP-2 at either low or high dosage on the bone-forming potential of constructs in a mice ectopic model. Our results showed that the addition of only low dose of BMP-2 was beneficial to enhance the bone-forming potential of MSCs, whereas high dose of BMP2 overcame the advantage of combining this growth factor with MSCs. Expressions of select genes of both murine and human origins in TE constructs demonstrated that the beneficial effect of low dose of BMP-2 with implanted human MSCs did not involve enhanced differentiation of these cells into osteoblasts or induction of paracrine cues but rather involved induction of the osteogenic differentiation of the host progenitors. Therefore, the advantage of combining BMP- 2 with MSCs to enhance the bone-forming potential of TE constructs appeared to be an additive effect of both components rather than a synergistic one.

Impact Statement: A strategy for improving the efficacy of stem cell-based bone tissue engineering (TE) constructs is to combine bone morphogenetic protein-2 (BMP-2) with multipotent stromal cells (MSC). Previous studies on the potential cooperative effect of BMP-2 with human multipotent stromal cells (hMSCs) on bone formation in vivo have, however, shown contradictory results likely due to the various and/or inappropriate BMP-2 doses. Our results provided evidence that the addition of BMP-2 at low dose only was beneficial to improve the osteogenic potential of hMSCs-containing TE constructs, whereas BMP-2 delivered at high dose overcame the advantage of combining this growth factor with hMSCs. This new knowledge will help in designing improved combination strategies for tissue regeneration with better clinical outcomes.

\section{INTRODUCTION:}

Stem cell-based bone tissue engineering (TE) approaches are currently developed by combining osteoprogenitors (such as multipotent stromal cells [MSC]) with a supporting substrate for the repair of large bone defects. The efficiency of bone TE in both experimental and clinical studies, however, remains unsatisfactory. Indeed, in large animal models, in which full bone regeneration was demonstrated using MSC-containing TE constructs, inconsistent bone repair outcomes have limited large-scale clinical applications.1-4 This evidence emphasizes the great need for improving the efficacy of bone TE constructs. Among the causes responsible for failure to bone regeneration, an insufficient osteoinductive potential of these cell-containing TE constructs has been implicated.2,5,6 To overcome this issue, the strategy of adding an osteoinductive cue to stem cells was addressed in the present study.

Bone morphogenetic protein-2 (BMP-2) is a potent osteoinductive growth factor involved in the early stage of bone formation and repair.7 The literature on the in vivo potential benefit of BMP-2 addition to stem cells (either bone marrow- or adipose-derived stem cells) on new bone formation or healing has been, however, controversial. In fact, some studies reported that such codelivery led to a significant increase in bone formation.8-12 In particular, a recent study published by our group demonstrated that a bone TE construct containing autologous MSCS combined with BMP-2 used to treat critical-sized defect in sheep fully repaired $50 \%$ of the tested animals as only $20 \%$ and $30 \%$ of the animals repaired when treated with either BMP-2 alone or MSCs alone, respectively. 12 In contrast, other studies showed no improvement in bone formation with codelivery of both BMP-2 and stem cells compared with results obtained with BMP-2 treatment alone.13-16 Definite comparisons of these results, however, are not possible because the source of MSCs, the material scaffolds, the implantation model, and the doses of BMP-2 used were different.

This latter point is critical as BMP-2 promotes bone formation in a dosedependent manner 17 and most of the aforementioned studies assessed a single dose of BMP-2. Besides, BMP-2 has an optimal dose for therapeutic success, and delivery of supraphysiological doses generated deleterious side effects, including excessive bone formation and immune responses.18,19 For these reasons, substantial work needs to be done (i) to determine the effective dosage of BMP-2, which promotes MSCmediated bone formation and (ii) to provide detailed mechanistic insights on the relation among BMP-2, MSCs, and bone formation at this minimal dosage. 
To this aim, we determined the effects of two BMP-2 dosages on the bone-forming potential of TE constructs containing human MSCs (hMSCs) and assessed the influence of BMP-2 on the host murine progenitor cells as well as on the direct and paracrine contribution of implanted hMSCs in a mice ectopic model.

\section{MATERIALS AND METHODS:}

\section{Material scaffolds}

Two hundred to $300 \mu \mathrm{m}$ calcium carbonate particles (Coral from Porites sp.; Biocoral ${ }^{\circledR}$, Inoteb, France) were used as the bioceramic scaffolds. These particles were sterilized by autoclaving at $120^{\circ} \mathrm{C}$ for $20 \mathrm{~min}$.

hMSC isolation and culture

HMSCs were harvested from bone marrow obtained as discarded tissue during routine bone surgery from three patients (one woman and two men, 15, 22, and 31 years of age, respectively) at the Lariboisiere Hospital (Paris, France). The tissues were collected with the respective donor's consent in agreement with Lariboisiere Hospital regulations. hMSCs were isolated from each donor bone marrow using a procedure adapted from literature reports 20 and were characterized for the expression of select CD markers (specifically, positive for CD90, CD73, CD105, and negative for CD45; data not shown). These cells were cultured in Alpha-Minimum Essential Medium (Sigma) containing $10 \%$ fetal bovine serum (PAA Laboratories) and antibiotics (PAA Laboratories) into tissue culture plates. Adherent cells from each donor were cultured separately until passage 3 and then pooled before seeding on coral scaffolds.

\section{Bone morphogenetic protein-2}

Recombinant human BMP-2 powder (InductOs ${ }^{\circledast} 12 \mathrm{mg}$; Medtronic, France) was reconstituted in sterile $1 \mathrm{mM} \mathrm{HCl}$ solution $(\mathrm{pH} \sim 3)$ at a concentration higher than $100 \mu \mathrm{g} / \mathrm{mL}$, aliquoted, and kept at $-80^{\circ} \mathrm{C}$ un I use. The initial BMP-2 concentration was assessed by UV detection at $280 \mathrm{~nm}$ (using a NanoDropTM1000 UV-Vis Spectrophotometer) with the extinction coefficient fixed at 1.41 absorbance units per $\mathrm{mg} / \mathrm{mL}$.

\section{Preparation of TE constructs}

Bare coral particles

Twenty-five milligrams of sterile coral particles were washed with complete culture medium.

\section{hMSC-containing coral particles}

Twenty-five milligrams of sterile coral particles were immersed in complete culture medium at $37^{\circ} \mathrm{C}$ for at least $1 \mathrm{~h}$. After the medium removal, $106 \mathrm{hMSCs}$ were seeded onto the particles and allowed to adhere in static condition at $37^{\circ} \mathrm{C}$ overnight.

\section{BMP-2-loaded coral particles}

Twenty-five milligrams of sterile coral particles were washed in $1 \mathrm{mM} \mathrm{HCl}$ and loaded with $50 \mu \mathrm{L}$ of BMP-2 diluted at either 10 or $100 \mu \mathrm{g} / \mathrm{mL}$ (defined as low dose [LD] and high dose [HD], respectively) in $1 \mathrm{mM} \mathrm{HCl}$ and maintained under mild shaking at $4^{\circ} \mathrm{C}$ overnight. The adsorption rates of BMP-2 onto coral particle were determined and found to be $81 \%$ and $84 \%$ for $L D$ and HD, respectively (Table 1 ). The retention capacities of these bioceramic scaffolds for BMP-2 were determined by monitoring the kinetics of protein release during $48 \mathrm{~h}$ (Supplementary Fig. S1A; Supplementary Data are available online at www.liebertpub.com/tea). Both dose cases displayed similar release profiles with an initial burst release of BMP-2 occurring during the first hour of the experiment (due to desorption of unbound growth factor) followed by a minimal (up to $3 \%$ ) BMP-2 released afterward. Maintenance of the biological activity of BMP2 coated onto coral particles was confirmed using pluripotent $\mathrm{C} 2 \mathrm{C} 12$ myoblasts as a cell model21 (Supplementary Fig. S1B). At similar doses of BMP-2, the bioactivity of BMP-2 adsorbed onto coral particles was slightly lower than the one of soluble BMP-2, but still significant.
Six different types of TE constructs were prepared by mixing at a 1:1 ratio the aforementioned types of particles in $8 \mathrm{~mm}$ wells (Table 1). Before mixing with other particles, BMP-2-loaded coral particles were quickly washed twice with culture medium (to neutralize the acidity). Before implantation, the mixed particles were embedded in $100 \mu \mathrm{L}$ fibrin gel (prepared using $18 \mathrm{mg} / \mathrm{mL}$ fibrinogen and $50 \mathrm{U} / \mathrm{mL}$ Thrombin; Tissucol ${ }^{\circledast}$, Baxter).

\section{In vivo implantation}

Eight-week-old, female, nude mice (RJ: NMRI-nu [nu/nu]) were obtained from JANVIER LABS and handled in accordance with the European Directive 2010/63/EU regarding the protection of animals used for scientific purposes. All animal experiments were performed following approval of the protocols by the Ethics Committee on Animal Research of Lariboisiere/Villemin (no. CEEA-LV/2014-10-20), Paris, France. Under general anesthesia of each animal, the TE constructs were randomly implanted subcutaneously into both the thoracic and abdominal dorsal regions on each side of the rachis. 22 The anesthesia protocol used included premedication with $0.05 \mathrm{mg} / \mathrm{kg}$ buprenorphine (Buprecare ${ }^{\circledR}$ $0.3 \mathrm{mg} / \mathrm{mL}$; Axience, France) $20 \mathrm{~min}$ before anesthesia (intraperitoneal injection of $10 \mathrm{mg} / \mathrm{kg}$ xylazine, Rompun ${ }^{\circledR} 2 \%$; Bayer HealthCare, France, and $100 \mathrm{mg} / \mathrm{kg}$ ketamine, Ketamine $1000^{\circledR}$; Mérial, France).

\section{Microcomputed tomography analysis, histology, histomorphometry, and immunohistochemistry}

At 8 weeks postimplantation, the mice were sacrificed (using pentobarbital, Dolethal ${ }^{\circledR}$; Vetoquinol) and the retrieved constructs ( $n=12$ per group) were immediately fixed in $10 \%$ neutral buffered formaldehyde solution for $24 \mathrm{~h}$. The fixed specimens were imaged and analyzed using a high-resolution microcomputed tomography $(\mu-\mathrm{CT})$ machine (Skyscan 1172; Skyscan, Aartselaar, Belgium) to determine the volume of the explanted TE constructs. The data were collected without a filter at $80 \mathrm{kV}$ and $100 \mu \mathrm{A}$ for $150 \mathrm{~ms}$. Visualization and reconstruction were obtained using NRecon software (Skyscan, Belgium). Because the explants displayed an oblate ellipsoid shape, the implant volumes were measured using the formula: Volume $=[4 \times \pi \times$ length $\times$ width $\times$ height $] / 3$.

Quantification of bone and coral volumes using $\mu$-CT were not possible because their respective gray-level thresholds did not provide conclusive discrimination. The constructs were, therefore, processed for undecalcified histology and histomorphometric analysis. The bone surface area (stained in red), coral surface area, and bone marrow-like tissue surface area were measured in each specimen section and expressed in $\mu \mathrm{m} 2$ according to established methods.22 The atomic chemical composition analysis of TE construct bone (and compared with a mouse cortical bone) was performed from resin-embedded sections using scanning electron microscopy (SEM)-energy-dispersive X-ray (EDX) spectroscopy using an Analytical UHR Schottky Emission Scanning Electron Microscope SU-70 (HITACHI).

A set of TE constructs ( $n=4$ per group) were implanted for 28 days, retrieved, fixed in $4 \%$ paraformaldehyde $(\mathrm{pH} 7.4)$, decalcified in ethylenediaminetetraacetic acid $(14.5 \% \mathrm{w} / \mathrm{v})$ at $4^{\circ} \mathrm{C}$ for 2 weeks, and embedded in paraffin. Some sequential sections were examined for the presence of human beta-2-microglobulin (a membrane protein that enables tracking human cells) immunodetection, using the Envision+ Kit (Dako) and polyclonal rabbit anti-beta-2-microglobulin (1/1000; Novocastra) as the primary antibody. 22

\section{Quantitative gene expression analysis by RT-PCR on explanted TE constructs}

Another set of TE constructs ( $n=6$ per group) were prepared and either nonimplanted (day 0) or implanted for 7 and 28 days, retrieved, and stored at $-80^{\circ} \mathrm{C}$. The frozen explanted constructs were then finely chopped with a scalpel blade, and total RNA was extracted by adding $1 \mathrm{~mL}$ of TRIzol ${ }^{\circledast}$ (Thermo Scientific). The RNA concentration and purity were 
determined using a NanoDrop spectrophotometer (NanoDrop 1000; LabTech). cDNA was obtained after reverse transcription of $3 \mu \mathrm{g}$ of purified RNA using the Superscript II enzyme (Life Technologies) and random primers. Quantitative PCR was then performed using 25 and $75 \mathrm{ng}$ cDNA in 96-well plates (iCycler iQ PCR plates; Bio-Rad) and TaqMan gene expression assays (Life Technologies) for mouse genes and human genes, respectively, following the manufacturer's instructions and using the MyiQ $^{\text {TM }}$ Single-Color Real-Time PCR Detection System (Bio-Rad). The results were normalized to those of the respective GAPDH as internal standard. The full names of the genes monitored and the assay IDs are given in Supplementary Table S1.

\section{Statistical analyses}

Statistical analyses were performed using a commercially available software package (GraphPad Prism, version 6.0c; GraphPad Software, Inc., La Jolla). Quantitative data were expressed as mean \pm SEM. The one-way analysis of variance (ANOVA) followed by Tukey's post hoc test was used to compare mean of more than two groups. The quantitative kinetics data were analyzed using two-ways ANOVA followed by Tukey's post hoc test For all analyses, differences at $p<0.05$ were considered as statistically significant.

\section{Results}

\section{In vivo osteogenic performance of TE constructs}

To determine the osteogenic potential of the constructs containing both the hMSCs and BMP-2, these specimens were implanted subcutaneously in the back of mice. The six conditions tested, including appropriate controls (specifically, constructs containing either bare coral or coral loaded with hMSCs or coral loaded with BMP-2 at either LD or HD) are listed in Table 1. Based on the determined adsorption rate, the total BMP2 dose delivered in vivo was estimated to be 0.4 and $4.2 \mu \mathrm{g}$ for the LD and $\mathrm{HD}$, respectively.

After 8 weeks, the hMSC-HD explanted constructs and their HD controls exhibited 1.6-2.4-fold larger volumes than the other constructs as determined by $\mu-C T$ analysis ( $p<0.05$ ) (Fig. 1A). Representative images from histology sections from each type of construct tested are shown in Figure 1B. Minimal new bone tissue was present both in the hMSC constructs and LD constructs, whereas no bone tissue was observed in the control coral constructs (Fig. 1Ba-c). More new bone tissue deposited around residual coral particles throughout the specimen was observed when hMSCs were combined with LD in constructs (Fig. 1Bd). In contrast, a bone tissue organized as a peripheral shell connected with thin internal trabeculae and enclosing bone marrow-like tissue, and few residual coral particles was found in hMSC-HD-containing constructs; the cell-free HD constructs exhibited same histological features (Fig. 1Be, f).

Examination under higher magnification of both hMSC- and hMSC-LDcontaining constructs revealed the presence of woven bone tissue with large nucleated cells and abundant osteoid encircled by bone-lining cells present all around the new bone tissue, illustrating its immature nature as well as high bone-forming activity (Fig. 2A, B). In contrast, newly formed bone tissue in the hMSCs-HD-containing constructs appeared more lamellar and mature (Fig. 2C). In the latter constructs, the peripheral bone tissue appeared still active with the presence of a layer of osteoid that is, however, much thinner than in the case of the hMSC- and hMSC-LDcontaining constructs (Fig. $2 \mathrm{C}$ ); besides, the inner trabeculae bone tissue appeared either less active or quite inactive with total absence of osteoblasts laying down new bone tissue (Fig. 2D). Noteworthy, these HDcontaining constructs were filled with bone marrow containing numerous adipocytes.

SEM-EDX analyses revealed that the $\mathrm{Ca} / \mathrm{P}$ ratios in bone tissue from all TE constructs were in the same range (from 1.92 to 1.98), with the exception of the hMSCs-HD-containing constructs, from which the $\mathrm{Ca} / \mathrm{P}$ ratio was lower (1.67) (Supplementary Fig. S2A); it is worth to notice that the $\mathrm{Ca} / \mathrm{P}$ ratio from mouse cortical bone was 1.5. While the $\mathrm{P}$ content was similar in all groups, the Ca content was lower in LD- and hMSCs-HD-containing constructs as well as in mouse cortical bone (Supplementary Fig. S2B). These data indicated that, with the exception of hMSCs-HD-containing constructs, the degrees of calcification of the ectopic induced bone tissue in TE constructs were similar and higher than the one of mature cortical bone.

Histomorphometric quantification confirmed the qualitative observations (Fig. 3):

Bone quantification indicated that the addition of the LD to hMSC-containing constructs significantly $(p<0.05)$ enhanced the new bone tissue compared with constructs containing hMSCs only. The addition of the HD to hMSC-containing constructs, however, induced an amount of bone dramatically higher than those induced by the other groups, except the control HD construct. Thus, in this case, the presence of hMSCs in the HD constructs was not beneficial and did not change the osteogenic outcome (Fig. 3A).

The bone marrow tissue quantification confirmed that constructs containing both hMSCs and LD or either one of these components did not contain bone marrow, whereas the hMSCs-HDcontaining constructs were substantially (up to $50 \%$ of the surface area) filled with this tissue (Fig. 3B). It is worth to notice that the absence of hMSCs in the HD constructs significantly $(p<0.05)$ increased the volume of the bone marrow. However, independently of the bone-marrowcontaining explant analyzed, the adipocyte extent did not change and comprised $47 \% \pm 21 \%$ of the bone marrow tissue (data not shown).

The extent of the scaffold material resorption in constructs was also quantified (Fig. 3C). All constructs containing BMP-2 exhibited levels of coral resorption significantly higher $(p<0.05)$ than that found with the control constructs of bare coral. Additionally, the absence of hMSCs in BMP-2-containing constructs enhanced resorption levels in comparison with the hMSC-containing constructs.

When comparing the efficacy of the two dosages, these results provided evidence that only low dose of BMP-2 enhances the osteogenic potential of hMSC-containing constructs compared with treatment with either hMSCs alone or BMP-2 alone. In contrast, at high dose, BMP-2 exhibited a strong bone-forming potential by itself, and its addition to hMSC constructs did not improve the osteogenic effect. For these reasons, the following studies, aiming at understanding the beneficial effect of BMP-2 on hMSC-containing constructs, were performed using BMP-2 at low dose only.

\section{Role of BMP-2 on codelivered MSCS in TE constructs}

Constructs containing both hMSCs and LD were specifically explanted at shorter postimplantation times and examined in comparison with control constructs containing either hMSCs or LD.

Immunostaining of human $\beta 2$-microglobulin enabled visualization of the implanted hMSCs still present in sections of the explanted constructs 28 days postim plantation (Fig. 4). Positive human cells of both the hMSC and hMSCs-LD constructs were detected as bundles in the fibrous tissue surrounding the coral granules and bone tissue (Fig. 4A, B). In both types of constructs, while most of the osteocytes were not labeled (at a higher extent in the hMSCs-LD constructs), some human cells were observed along the periphery of the coral particles and embedded in osteocyte lacunae (arrows in Fig. $4 \mathrm{~A}-\mathrm{C}$ ); these results provided evidence that the new bone tissue was partly formed by the murine host cells and partly by the human implanted cells.

To further characterize the relative implication of mouse and human osteoprogenitor cells in the newly formed bone tissue, kinetics analysis of the both expressed murine (Fig. 5) and human (Fig. 6) osteogenesisrelated genes in TE constructs explanted at 0,7 , and 28 days postimplantation were performed. The expressions of the mRUNX2, mIBSP, mbGLAP, and mSPP1 genes increased with the implantation time 
(Fig. 5). Interestingly, in comparison with coral-, hMSC-, and LD-containing constructs, expression of $\mathrm{mRUNX} 2, \mathrm{mIBSP}$, and mbGLAP were significantly upregulated in the hMSC-LD-containing constructs (Fig. 5). Expression of mSPP1, a known regulator of mineral deposition in the bone extracellular matrix, was similar in all types of constructs (Fig. 5). Similarly, the expressions of human osteogenesis-related genes (i.e., hRUNX2, hIBSP, hbGLAP, and hSPP1) also increased with the implantation time (Fig. 6A), confirming the osteogenic differentiation of the surviving implanted human MSCs. No difference, however, was found in the expression of these genes in both hMSC-LD and hMSC-containing constructs at each postimplantation time tested.

To assess the paracrine effect of implanted human MSCs on host murine progenitors, gene expressions of hSDF1 and hPDGF-B (known for being involved in hMSC recruitment) as well as of hVEGF2 (known for its involvement in angiogenesis) were analyzed (Fig. 6B). Expressions of these three genes were upregulated at day 7 and downregulated at day 28 postimplantation. Only the expression of hPDGF-B was found significantly higher (5-fold) in hMSCS-LD constructs than in hMSC constructs at day 7 postimplantation.

The aforementioned data provided evidence that the higher bone formation observed with hMSCs-LD constructs was most probably due to higher murine osteogenic differentiation; moreover, the effect of BMP-2 at low dose to either induce the differentiation of the grafted human MSCs into osteoblasts or modulate their paracrine cues was null or minimal.

\section{DISCUSSION:}

In the present study, we assessed the advantage of combining BMP-2, a potent osteoinductive growth factor involved in the early stage of bone formation, with hMSCs to enhance the bone-forming potential of TE constructs in an ectopic mouse model; more specifically, we determined its effective dosage that improves the osteogenic potential of hMSCcontaining constructs. Our results provided evidence that the addition of high dose of BMP-2 induced a bone tissue in higher amount and more mature than those induced by low dose BMP-2; its codelivery with hMSCs, however, did not improve the osteogenic potential of constructs. In contrast, a significant benefit of BMP-2, combined with hMSCs on the bone-forming potential of these constructs, was found in the presence of a low dose of BMP-2. This effect seems to be due to BMP-mediated induction of the osteogenic differentiation of the host progenitors only and not of the grafted human cells.

Several previous studies have tested the potential cooperative effect of BMP-2 with MSCs on bone formation in both ectopic and orthotopic site with contradictory results. While some studies reported that such codelivery led to a significant increase in bone formation,8-11,23 other studies showed no improvement compared with results obtained with BMP-2 treatment alone.13-16 These inconsistent outcomes are likely due to the various and/or inappropriate doses of BMP-2 used in these studies. In fact, determining the accurate dosage of BMP-2 in association with MSCs is critical since BMP-2 induces bone formation in a dose-dependent manner17 and its delivery in high doses has been reported to be associated with adverse effects, including excessive bone formation, ectopic bone formation, inflammatory responses, osteoclast-mediated bone resorption, and inappropriate adipogenesis.18,24 In the present study, hMSCs were combined with BMP-2 at either low $(10 \mu \mathrm{g} / \mathrm{mL})$ or high $(100 \mu \mathrm{g} / \mathrm{mL})$ dose (corresponding to 0.4 and $4.2 \mu \mathrm{g}$ per TE construct, respectively) to determine the effective BMP-2 dosage that enhances the intrinsic osteogenic potential of implanted hMSCs. It should be noted that the high dose used in the present study was lower than those that induce adverse side effects in rats (more than $300 \mu \mathrm{g} / \mathrm{mL}$ ). 19

The osteogenic potential of the TE constructs was assessed in vivo in an ectopic mouse model, which has the advantage of evaluating the "true" osteogenic capacity of constructs since osteoconduction from the bone bed of the recipient animals does not occur at this location. Implantation of bone TE constructs in "unphysiological" ectopic site does not, however, enable the evaluation of high-dose BMP-2-mediated adverse effects. As expected, the amount of newly formed bone enhanced with the amount of implanted BMP-2 and this outcome was also observed in the presence of hMSCs.

When used at a high dose, the strong bone-forming potential of BMP-2 largely superseded the hMSC-mediated osteogenic outcome, and, therefore, addition of BMP-2 within hMSC-containing constructs had no additive effect on bone formation. Compared with the other constructs tested, the addition of high dose of BMP-2 along with or without hMSCs, led to larger-sized constructs. Histological analysis revealed that these constructs induced large bony ossicles, typical of BMP-2-induced ectopic bone induction with thin and mature bone trabeculae present mainly at the outer part of the implants forming a shell, and fatty bone marrow occupying the center of the ossicles. Additionally, weak or lacking boneforming activity was observed in these constructs.

Interestingly, codelivery of hMSCs with high dose of BMP-2, although not changing the amount of newly formed bone, significantly decreased the size of the explanted TE construct, limited coral resorption, reduced the amounts of fatty bone marrow, and promoted a bone tissue with a calcification degree comparable to those of murine cortical bone, suggesting that the presence of hMSCs modulates the excessive biological effects of high-dose BMP-2. Similarly, in a previous study, Keibl et al. noticed that the association of hMSCs to BMP-2 significantly reduces the periosteal callus volume in a femoral transcortical defect in rat, whereas observed in excess with BMP-2 alone.16 Further studies, however, are necessary to decipher the mechanism of action of hMSCs on the high-dose BMP-2-mediated excessive biological effects.

While BMP-2 delivered at high dose overcame the advantage of combining this growth factor with hMSCs, in contrast, when used at low dose, the BMP-2 combined with hMSCs significantly enhanced the boneforming potential of the TE constructs in comparison with constructs containing either hMSCs or BMP-2 only. This observation was in accordance with a recent study published by our group that provided evidence that the treatment of a critical-sized defect in sheep with codelivery of BMP-2 at low dose $(10 \mu \mathrm{g} / \mathrm{mL})$, autologous bone marrowderived MSCs and coral particles improved, but not significantly, the bone healing in comparison with that mediated by single treatment of either MSCs or low-dose BMP-2.12 In the present study, the ectopic newly formed bone (specifically, aspect and high activity with great extent of osteoid and bone-lining osteoblasts as well as degree of calcification) appeared similar to the one induced by hMSCs alone.

We next sought to determine the role of BMP-2 codelivered with hMSCs since it remains poorly understood so far. It is reported that the ectopic bone formation mediated by BMP-2 results from osteogenic differentiation of circulating mesenchymal precursor cells (including myoblast 21 and adipocyte8,25 progenitors), which are locally exposed to the growth factor. In the present study, we wondered whether BMP-2 also enhanced the osteogenic potential of the implanted hMSCs resulting in a synergized effect on the bone formation. Because high dose of BMP-2 combined with hMSC did not exhibit beneficial effect on the bone-forming potential compared with BMP-2 alone, these analyzes were performed on BMP-2 codelivered at low dose only.

Immunostaining of the implanted human cells provided evidence that both murine and human cells were present in the osteocyte lacunae proving that the new bone tissue was hybrid, chiefly formed by the murine host cells, but also formed by the human implanted cells (the later ones being present at a low extent). 


\section{Decambron et al}

Expressions of the select osteogenesis-related genes of both murine and human origins in TE constructs increased with the implantation time, confirming the osteogenic differentiation of the host murine progenitors along with the surviving implanted hMSCs. However, expressions of the select murine osteogenesis-related genes in TE constructs tested were upregulated (except, SPP1, a regulator of mineral deposition) in the presence of hMSCs combined with low-dose BMP-2, compared with their respective expressions in the presence of either one of these components alone. In contrast, expressions of osteogenesis-related genes of human origin were similar in TE constructs containing hMSCs either with or without low-dose BMP-2. Additionally, because in stem cell-based TE, hMSCs play not only a direct role on new tissue formation, but also an indirect role by supporting resident progenitor cells through paracrine mechanisms, 26 expression of key chemotactic and angiogenic factors in human MSCs was assessed. Only PDGF-B expression was found slightly higher in human MSCs in the presence of low-dose BMP-2, whereas those of SDF-1 and VEGF-A were similar in both types of constructs.

Taken altogether, these results suggested that whether the addition of low-dose BMP-2 to hMSC-containing construct promoted the host murine osteogenic differentiation, by contrast, its effect on implanted human MSCs toward either differentiation into osteoblasts or induction of paracrine cues was null or minimal. Therefore, the advantage of combining BMP-2 with hMSCs to enhance the bone-forming potential of hMSCs-containing TE constructs appeared to be an additive effect of both components rather than a synergistic one

\section{CONCLUSION}

The optimal bone repair and regeneration is a complex process that requires suitable scaffold, cells, and osteoinductive signals. Whether bone growth is promoted by the presence of either hMSCs or BMP-2, their combination is an attractive strategy to enhance the bone-forming potential of TE constructs. Results of the present study showed that the addition of low dose of BMP-2 was beneficial to improve the osteogenic potential of hMSCs containing TE constructs, whereas BMP-2 delivered at high dose overcame the advantage of combining this growth factor with hMSCs. Furthermore, this study demonstrated that this beneficial effect of low dose of BMP-2 with implanted hMSCs did not involve higher differentiation of these cells into osteoblasts or induction of paracrine cues. The knowledge gained from these evaluations will significantly help in designing improved combination strategies for tissue regeneration with better clinical outcomes.

\section{ACKNOWLEDGMENTS}

The authors thank Medtronic, Inc. for kindly providing BMP-2, Prof R. Bizios for reading the article, $\operatorname{Dr} M$. Bensidhoum for his help in $\mu$-CT analysis, and F. Pillier (UPR15-CNRS-LISE) for SEM-EDX analyses. This study was supported by the Centre National de la Recherche Scientifique (CNRS) and the Fondation pour l'Avenir (project $n^{\circ}$ ET3 704, 2013)

\section{Disclosure Statement}

The authors declare no competing financial interests

\section{References}

1. Bensaïd W, Oudina K, Viateau V, Potier E, Bousson V, Blanchat C, et al. De Novo Reconstruction of Functional Bone by Tissue Engineering in the Metatarsal Sheep Model. Tissue Eng. 11(5-6), 814, 2005.

2. Manassero M, Viateau V, Deschepper M, Oudina K, Logeart-Avramoglou D, Petite $\mathrm{H}$, et al. Bone Regeneration in Sheep Using Acropora Coral, a Natural Resorbable Scaffold, and Autologous Mesenchymal Stem Cells. Tissue Eng. Part A. 19(13-14), 1554,2013
3. Petite $H$, Viateau V, Bensaïd W, Meunier A, de Pollak C, Bourguignon M, et al. Tissue-engineered bone regeneration. Nat. Biotechnol. 18(9), 959, 2000.

4. Viateau V, Guillemin G, Bousson V, Oudina K, Hannouche D, Sedel L, et al. Longbone critical-size defects treated with tissue-engineered grafts: A study on sheep. J. Orthop. Res. 25(6), 741, 2007.

5. Amini AR, Laurencin CT, Nukavarapu SP. Bone tissue engineering: recent advances and challenges. Crit. Rev. Biomed. Eng. 40(5), 2012.

6. Dumic-Cule I, Pecina M, Jelic M, Jankolija M, Popek I, Grgurevic L, et al. Biological aspects of segmental bone defects management. Int. Orthop. 39(5), 1005, 2015.

7. Tsuji K, Bandyopadhyay A, Harfe BD, Cox K, Kakar S, Gerstenfeld L, et al. BMP2 activity, although dispensable for bone formation, is required for the initiation of fracture healing. Nat. Genet. 38(12), 1424, 2006

8. Mehrkens A, Saxer F, Güven S, Hoffmann W, Müller AM, Jakob M, et al. Intraoperative engineering of osteogenic grafts combining freshly harvested, human adipose-derived cells and physiological doses of bone morphogenetic protein-2. Eur. Cell. Mater. 24, 308, 2012.

9. Gohil SV, Adams DJ, Maye P, Rowe DW, Nair LS. Evaluation of rhBMP-2 and bone marrow derived stromal cell mediated bone regeneration using transgenic fluorescent protein reporter mice. J. Biomed. Mater. Res. A. 102(12), 4568, 2014.

10. Hou Rui, Chen Fulin, Yang Yaowu, Cheng Xiaobing, Gao Zhan, Yang Hongwei Ou, et al. Comparative study between coral-mesenchymal stem cells-rhBMP-2 composite and auto-bone-graft in rabbit critical-sized cranial defect model. J. Biomed. Mater. Res. A. 80A(1), 85, 2006.

11. Kim B-S, Choi M-K, Yoon J-H, Lee J. Evaluation of bone regeneration with biphasic calcium phosphate substitute implanted with bone morphogenetic protein 2 and mesenchymal stem cells in a rabbit calvarial defect model. Oral Surg. Oral Med. Oral Pathol. Oral Radiol. 120(1), 2, 2015.

12. Decambron A, Fournet A, Bensidhoum M, Manassero M, Sailhan F, Petite $H$, et al. Low-dose BMP-2 and MSC dual delivery onto coral scaffold for critical-size bone defect regeneration in sheep. J. Orthop. Res. 35(12), 2637, 2017

13. Boos AM, Loew JS, Deschler G, Arkudas A, Bleiziffer O, Gulle H, et al. Directly auto-transplanted mesenchymal stem cells induce bone formation in a ceramic bone substitute in an ectopic sheep model. J. Cell. Mol. Med. 15(6), 1364, 2011.

14. Fauzi Kamal A, Hadisoebroto Dilogo I, Untung Hutagalung E, Iskandriati D, Susworo R, Chaerani Siregar N, et al. Transplantation of mesenchymal stem cells, recombinant human BMP-2, and their combination in accelerating the union after osteotomy and increasing, the mechanical strength of extracorporeally irradiated femoral autograft in rat models. Med. J. Islam. Repub. Iran. 28, 129, 2014.

15. Herberg S, Aguilar-Perez A, Howie RN, Kondrikova G, Periyasamy-Thandavan S, Elsalanty ME, et al. Mesenchymal stem cell expression of SDF-1 $1 \beta$ synergizes with BMP-2 to augment cell-mediated healing of critical-sized mouse calvarial defects. J. Tissue Eng. Regen. Med. 11(6), 1806, 2017.

16. Keibl C, Fügl A, Zanoni G, Tangl S, Wolbank $S$, Redl $H$, et al. Human adipose derived stem cells reduce callus volume upon BMP-2 administration in bone regeneration. Injury. 42(8), 814, 2011.

17. Wang EA, Rosen V, D'Alessandro JS, Bauduy $M$, Cordes $P$, Harada $T$, et al. Recombinant human bone morphogenetic protein induces bone formation. Proc. Natl. Acad. Sci. 87(6), 2220, 1990.

18. James AW, LaChaud G, Shen J, Asatrian G, Nguyen V, Zhang X, et al. A Review of the Clinical Side Effects of Bone Morphogenetic Protein-2. Tissue Eng. Part B Rev. 22(4), 284, 2016.

19. Zara JN, Siu RK, Zhang X, Shen J, Ngo R, Lee M, et al. High Doses of Bone Morphogenetic Protein 2 Induce Structurally Abnormal Bone and Inflammation In Vivo. Tissue Eng. Part A. 17(9-10), 1389, 2011. 


\section{Decambron et al.}

20. Friedenstein AJ, Piatetzky-Shapiro II, Petrakova KV. Osteogenesis in transplants of bone marrow cells. Development. 16(3), 381, 196.

21. Katagiri T, Yamaguchi A, Komaki M, Abe E, Takahashi N, Ikeda $\mathrm{T}$, et al. Bone morphogenetic protein-2 converts the differentiation pathway of $\mathrm{C} 2 \mathrm{C} 12$ myoblasts into the osteoblast lineage. J. Cell Biol. 127(6 Pt 1), 1755, 1994.

22. Monfoulet L-E, Becquart $P$, Marchat $D$, Vandamme $K$, Bourguignon $M$, Pacard $E$, et al. The $\mathrm{pH}$ in the Microenvironment of Human Mesenchymal Stem Cells Is a Critical Factor for Optimal Osteogenesis in Tissue-Engineered Constructs. Tissue Eng. Part A. 20(13-14), 1827, 2014

23. Gohil SV, Kuo C-L, Adams DJ, Maye P, Rowe DW, Nair LS. Evaluation of the dono cell contribution in rhBMP-2 mediated bone formation with chitosan thermogels using fluorescent protein reporter mice. J. Biomed. Mater. Res. A. 104(4), 928, 2016.

24. Carragee EJ, Hurwitz EL, Weiner BK. A critical review of recombinant human bone morphogenetic protein- 2 trials in spinal surgery: emerging safety concerns and lessons learned. Spine J. 11(6), 471, 2011.

25. Chen D, Ji X, Harris MA, Feng JQ, Karsenty G, Celeste AJ, et al. Differential roles for bone morphogenetic protein (BMP) receptor type IB and IA in differentiation and specification of mesenchymal precursor cells to osteoblast and adipocyte lineages. J. Cell Biol. 142(1), 295, 1998

26. Baraniak PR, McDevitt TC. Stem cell paracrine actions and tissue regeneration Regen. Med. 5(1), 121, 2010

Corresponding author:

Delphine Logeart-Avramoglou, PhD

B2OA UMR 7052

Faculty of Medicine

University Denis Diderot

10 Avenue de Verdun

75010 Paris, France

E-mail: delphine.logeart@cnrs.fr

Received: January 14, 2018

Accepted: September 30, 2018

Online Publication Date: November 9, 2018

How to cite this article: Decambron A, Devriendt N, Larochette $\mathrm{N}$, et al.

Effect of the Bone Morphogenetic Protein-2 Doses on the Osteogenic

Potential of Human Multipotent Stromal Cells- Containing Tissue

Engineered Constructs. Tissue Eng. 2019;642-51.

https://doi.org/10.1089/ten.tea.2018.0146 
Decambron et al.

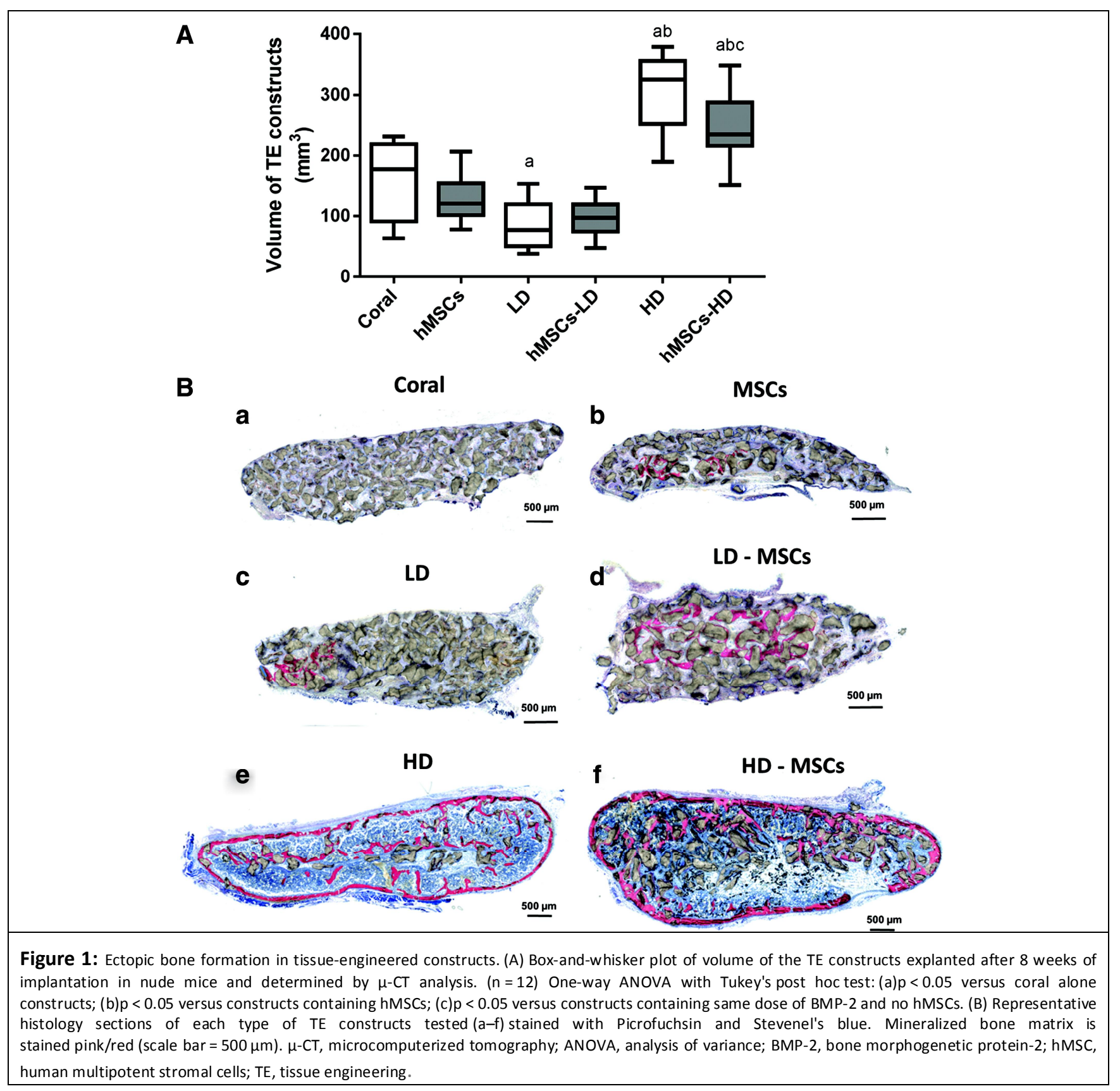




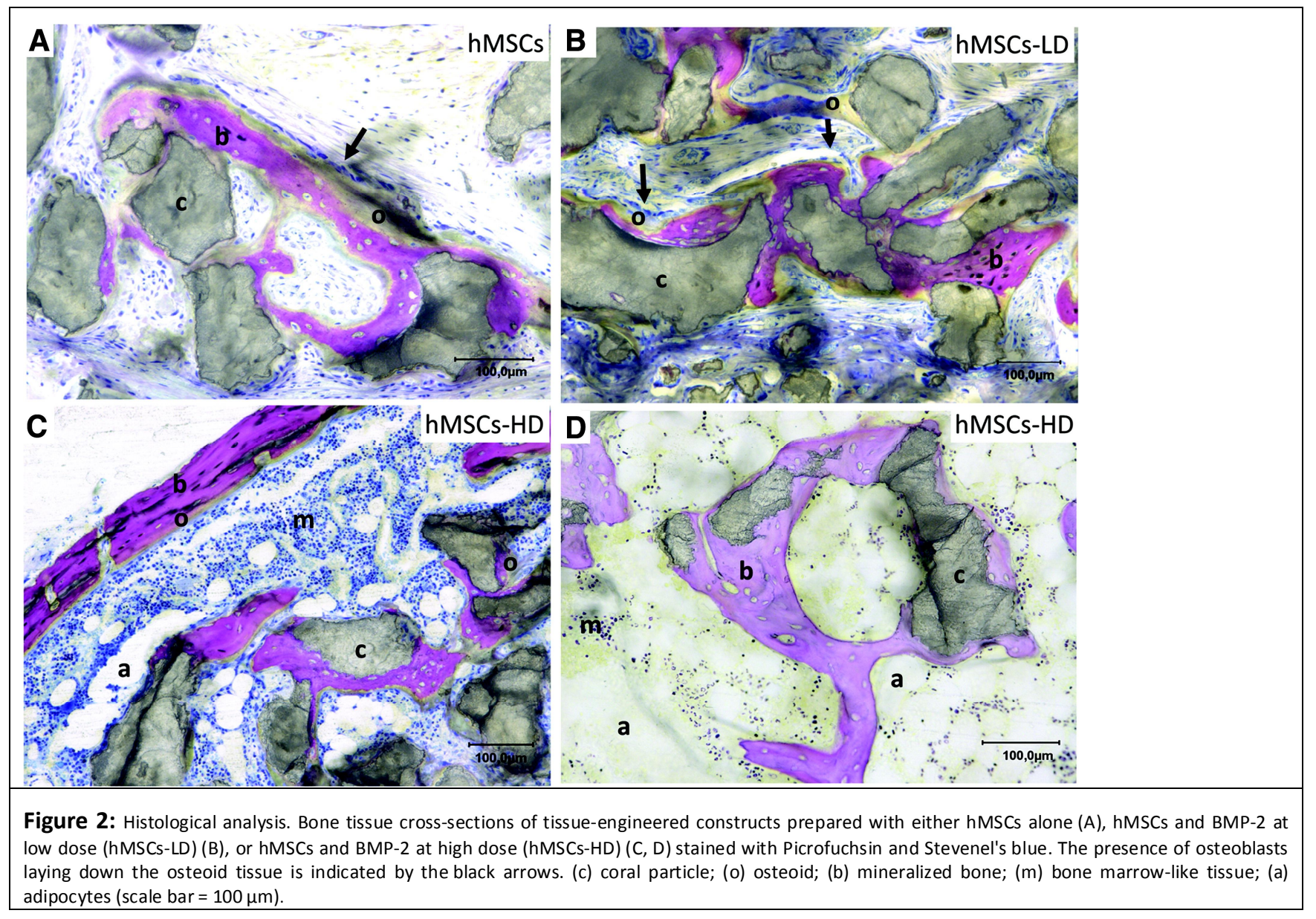


Decambron et al.

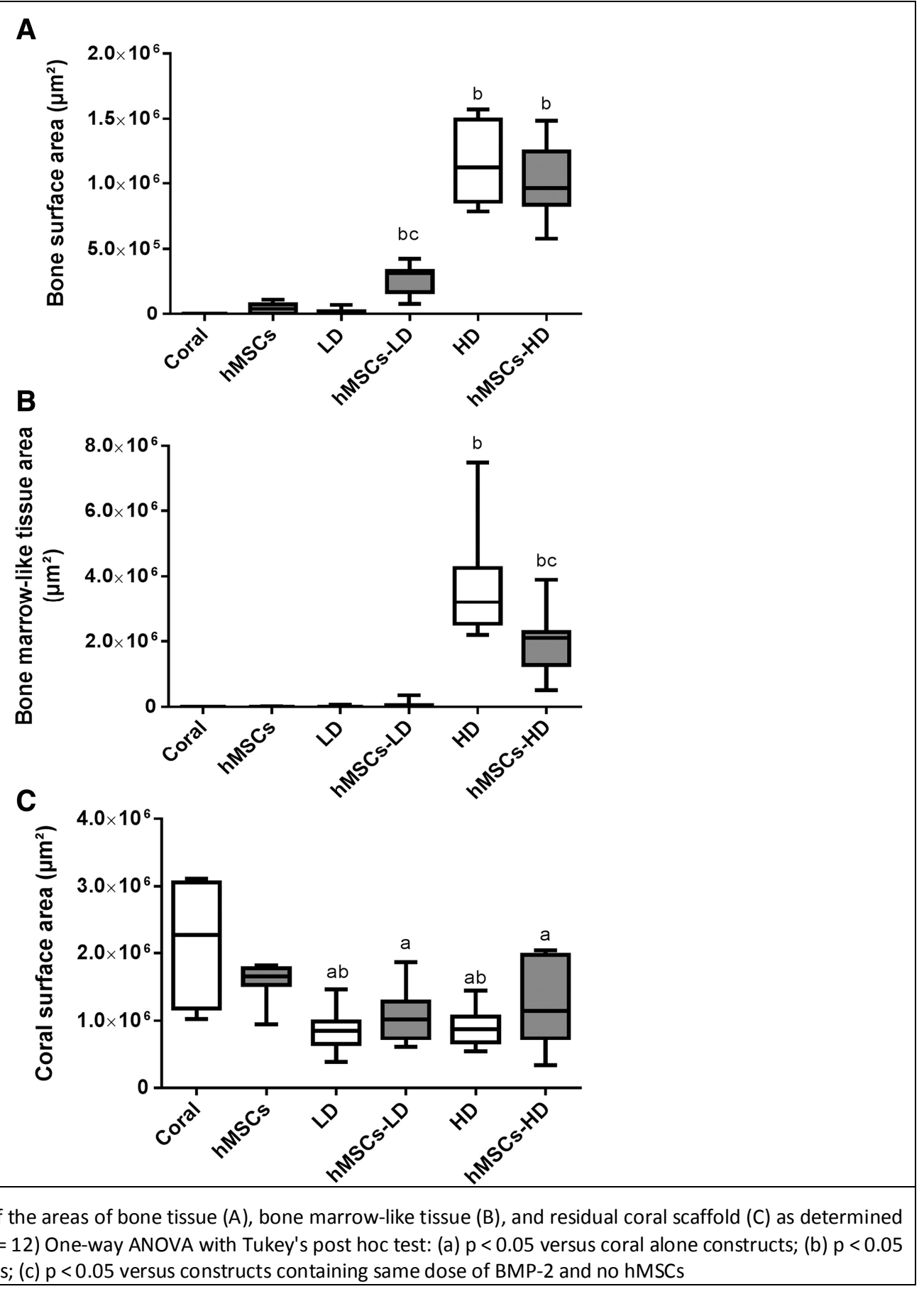

Figure 3: Box-and-whisker plots of the areas of bone tissue (A), bone marrow-like tissue (B), and residual coral scaffold (C) as determined by histomorphometric analysis. $(n=12)$ One-way ANOVA with Tukey's post hoc test: (a) $p<0.05$ versus coral alone constructs; (b) $p<0.05$ versus constructs containing hMSCs; (c) $p<0.05$ versus constructs containing same dose of BMP-2 and no hMSCs 


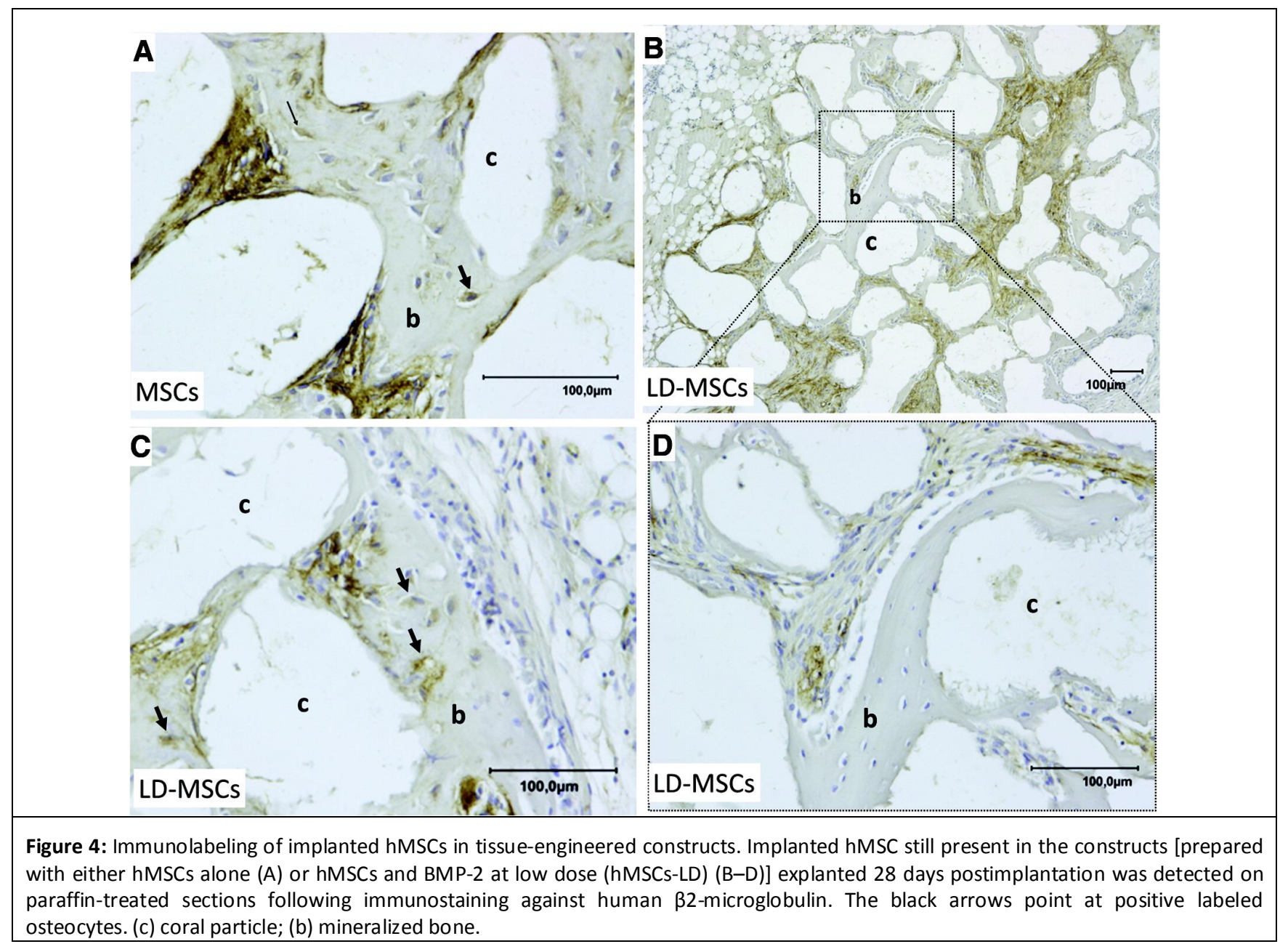




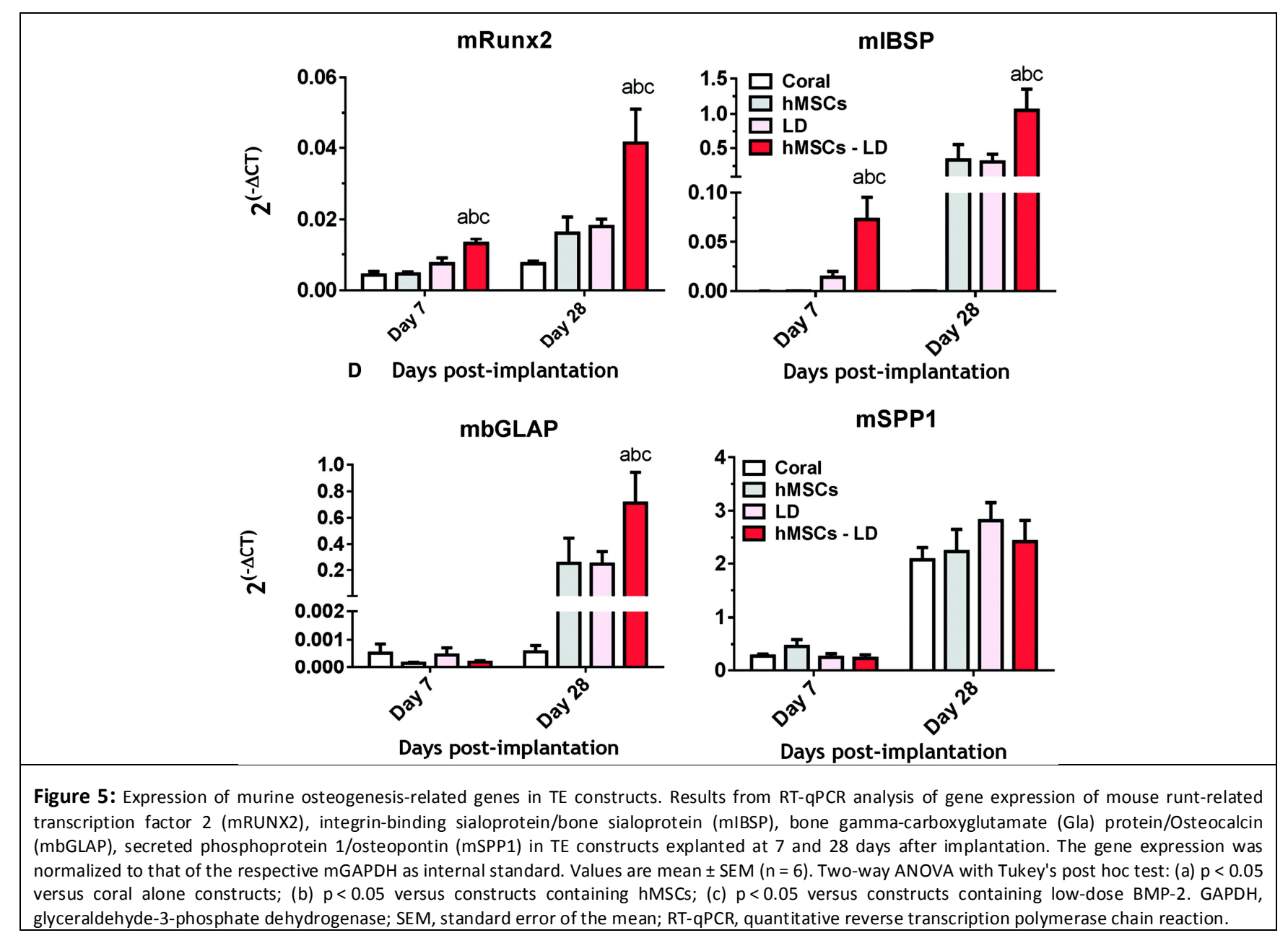




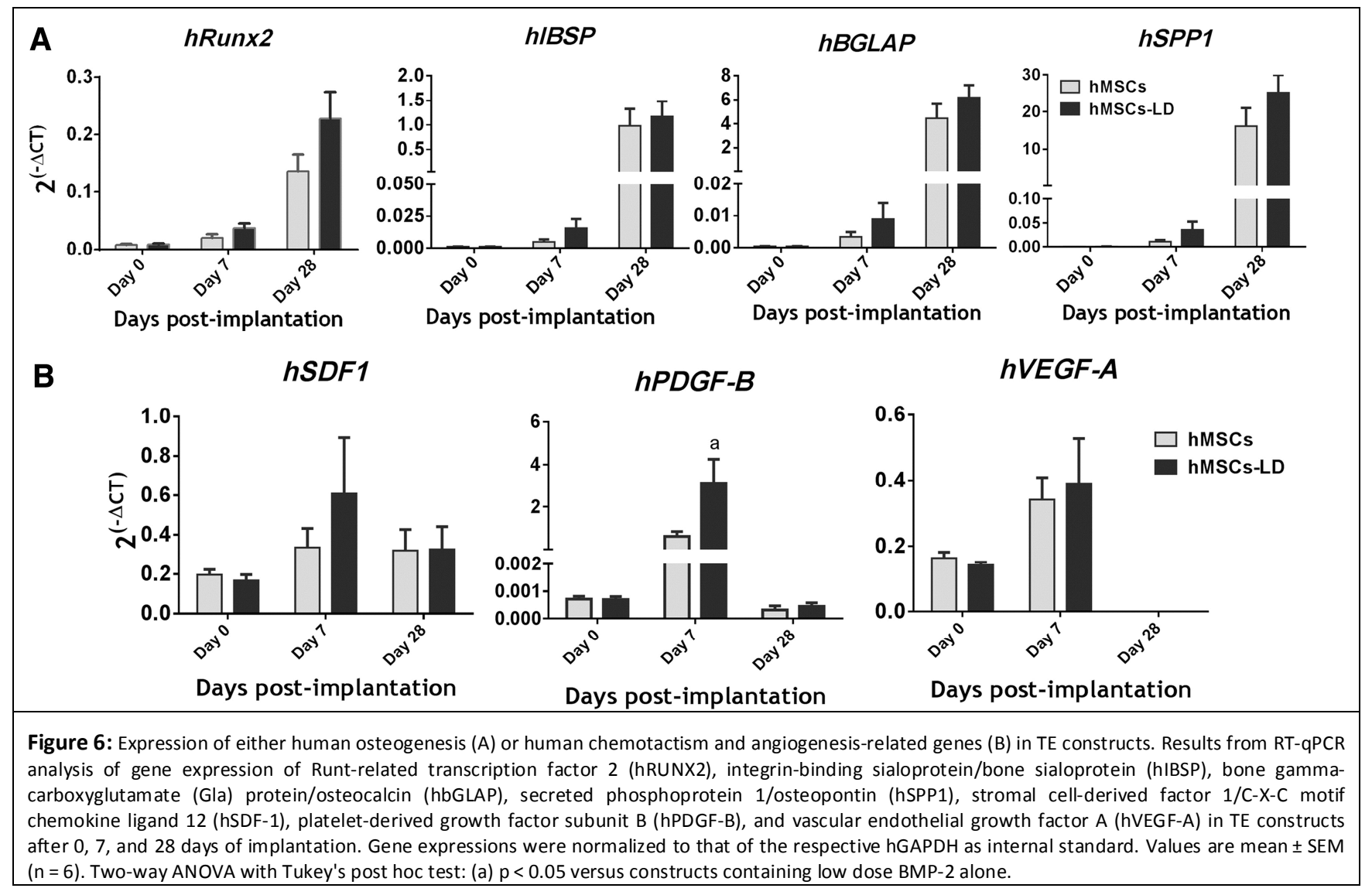


A

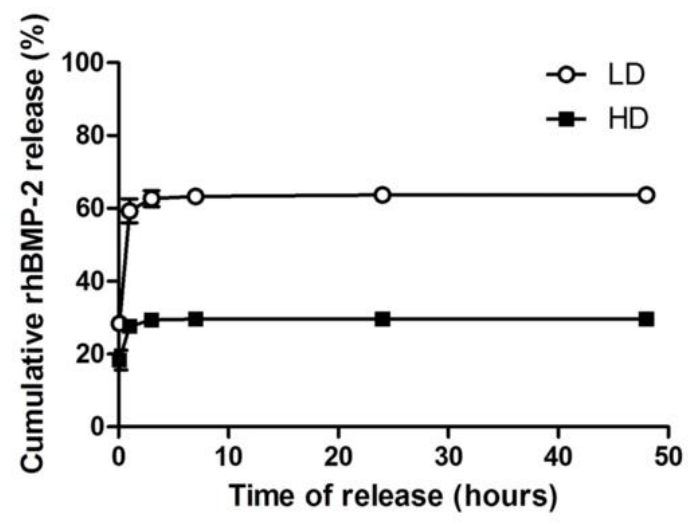

B

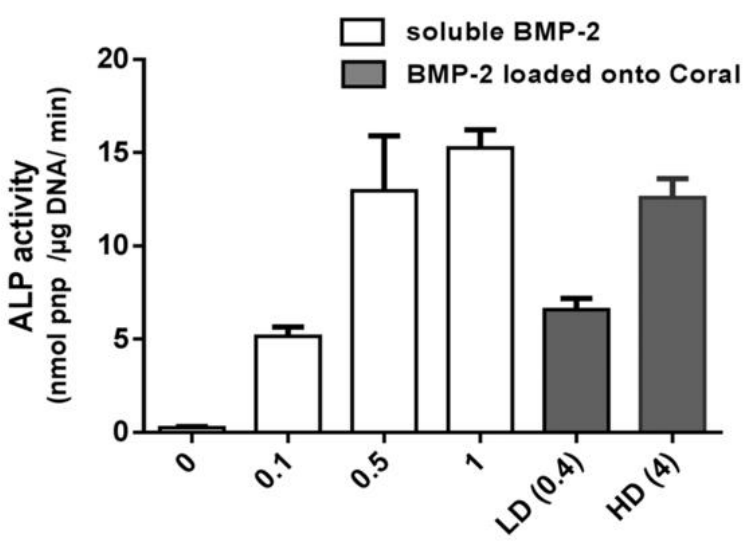

BMP-2 dose $(\mu \mathrm{g} / \mathrm{ml})$

Figure S1: $(A) \quad$ Kinetics of in vitro BMP-2 release from coral particles during 48 hours. Fifty mg of coral particles were coated with BMP-2 as described in the "Preparation of TE constructs" paragraph in the "Materials and Methods" section. Following removal of the non-adsorbed BMP-2 solution, the particles were rinsed, immersed in $1 \mathrm{~mL}$ of PBS $(0.15 \mathrm{M} \mathrm{NaCl})$ at $\mathrm{pH} 7.4 \mathrm{containing} 0.5 \% \mathrm{BSA}$ and $0.02 \%$ NaN3 and kept at $37^{\circ} \mathrm{C}$ under gentle shaking. At 1, 3, 7, 24 and $48 \mathrm{~h}$, the supernatants (containing the released BMP-2) were collected and replaced by $1 \mathrm{~mL}$ fresh buffer. The respective supernatants were aliquoted and stored at $-80^{\circ} \mathrm{C}$ until BMP-2 quantification was performed using an ELISA assay kit (DuoSet? ELISA Development System, R\&D Systems, MN) according to the manufacturer's protocol. The amount of released BMP-2 in each supernatant was expressed as a percentage of the initial adsorbed amount of BMP-2. Values are means \pm SEM from three separate experiments.

(B) Biological activity of the BMP-2 retained onto the coral particles. The bioactivity was determined by assaying the BMP-2induced alkaline phosphatase (ALP) activity (a marker of osteogenic differentiation) on murine C2C12 skeletal myoblasts (American Type Culture Collection) (Katagiri, J Cell Biol, 1994). C2C12 cells (100,000 in DMEM (Dulbecco's modified Eagle's medium) supplemented with 2 $\%$ FBS) were seeded on the particles (previously coated with BMP-2 and rinsed) and cultured under standard conditions. At day 6, the cells were rinsed with PBS and lysed using $200 \mu \mathrm{L}$ of lysis-buffer ( NaHCO3 $0.1 \mathrm{M}, \mathrm{Na} 2 \mathrm{CO} 30.1 \mathrm{M}, \mathrm{MgCl} 21 \mathrm{mM}$, Triton X-100 0.1 \%, pH 10.2) and freeze/thawed three times. The ALP activity in each lysate was determined as previously described (Degat et al., J Biomed Mate Res, 2009) and was normalized to the DNA content of the respective cell population using the PicoGreen kit (Thermo scientific). The level of ALP activity induced by the coral-adsorbed BMP-2 was compared to that obtained from the soluble BMP-2 (at $0.1,0.5$ and $1 \mu \mathrm{g} / \mathrm{ml}$ ) in the culture medium (positive controls). Values in parentheses are the estimated dose of BMP-2. Values are means \pm SEM from three separate experiments. 
Decambron et al.

A

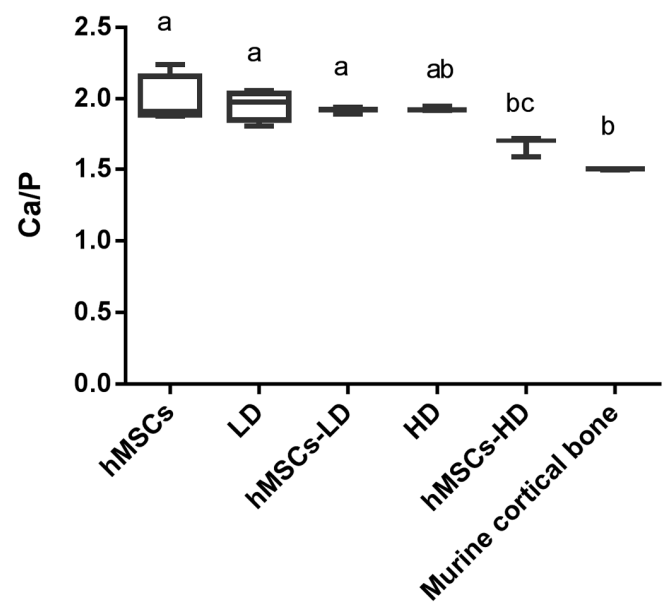

B

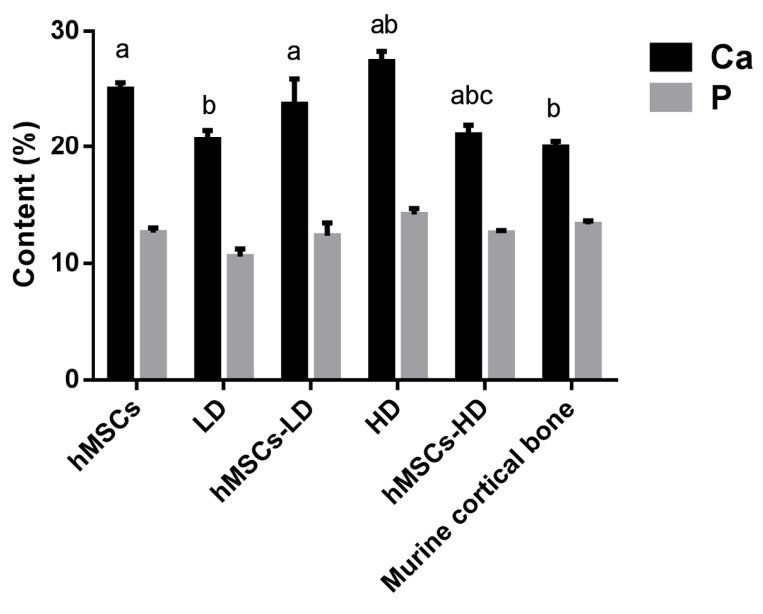

Figure S2: (A) Ca/P molar ratios and (B) Atomic Calcium and Phosphorus contents (\%) obtained from energy-dispersive X-ray point analysis from resin-embedded sections of TE-construct bone tissue and compared to a mouse cortical bone. Values are means \pm SEM from three separate analyses. One-way ANOVA with Tukey's posthoc test: (a) $p<0.05$ vs mouse cortical bone; (b) $p<0.05$ vs constructs containing hMSCs; (c) p < 0.05 vs constructs containing same dose of BMP-2 and no hMSCs.

Table 1: Experimental design of the TE constructs tested in vivo: Each TE construct used as implant contained 50 mg of coral particles prepared by mixing (1:1) particles either bare (Coral) or containing hMSCs or loaded with either low (LD) or high (HD) doses of BMP-2.

\begin{tabular}{|c|c|c|c|c|c|}
\hline TE constructs & MSCs & \multicolumn{4}{|c|}{ BMP-2 } \\
\hline & $\begin{array}{c}\text { Initial } \\
\text { concentration } \\
(\mu \mathrm{g} / \mathrm{mL})\end{array}$ & $\begin{array}{c}\text { Loaded } \\
\text { amount }(\mu \mathrm{g})\end{array}$ & $\begin{array}{c}\text { Adsorption } \\
\text { rate }(\%)^{\text {a }}\end{array}$ & $\begin{array}{c}\text { Implanted } \\
\text { amount }(\mu \mathrm{g})^{\mathrm{b}}\end{array}$ \\
\hline Coral & - & - & & & \\
hMSC & $10^{6}$ & - & 0.5 & $81 \pm 14$ & 0.4 \\
LD & - & 10 & 0.5 & $81 \pm 14$ & 0.4 \\
hMSCS - LD & $10^{6}$ & 10 & 5.0 & $84 \pm 4$ & 4.2 \\
HD & - & 100 & 5.0 & $84 \pm 4$ & 4.2 \\
\hline
\end{tabular}

a: The absorption rate of BMP-2 was calculated according to the equation: Adsorption rate (\%) $=([\mathrm{BMP}-2$ loaded - BMP-2 nonadsorbed]/BMP-2 loaded) $\times 100$. The amounts of loaded BMP-2 and non-absorbed BMP-2 were quantified using UV-detection. ${ }^{b}$ : The estimated amount of BMP-2 implanted in vivo was calculated by multiplying the loaded amounts by the adsorption rates. 